ARTICULO ORIGINAL

\title{
Frecuencia de enteroparásitos en niños y niñas del primer ciclo de la educación escolar básica de Escuelas Públicas de Ciudad del Este, Paraguay
}

\section{Enteroparasites frequency in children of the first cycle of basic education of Public Schools in Ciudad del Este, Paraguay}

\author{
*Cardozo Ocampos GE ${ }^{\mathrm{I}}$, Cañete Duarte $\mathbf{Z}^{\mathrm{I}}$, Lenartovicz $\mathbf{V}^{\mathrm{II}}$
}

IUniversidad Nacional del Este. Escuela de Posgrado, Universidad Católica Nuestra Señora de la Asunción-Alto Paraná. Facultad de Ciencias de la Salud, Paraguay

IIUniversidade Estadual do Oeste do Paraná (UNIOESTE). Cascavel, Brasil

\section{RESUMEN}

Las enfermedades parasitarias tienen amplia distribución mundial, y a pesar de los esfuerzos de los organismos de salud, siguen siendo un problema especialmente en los países en vias de desarrollo como el nuestro. El objetivo del presente trabajo, debidamente evaluado por la Comisión Nacional para el Desarrollo de la Bioética en Paraguay, fue la de determinar la tasa de frecuencia de enteroparásitos en niños y niñas del primer ciclo de la Educación Escolar Básica de Ciudad del Este (Paraguay). En el periodo de setiembre a diciembre del año 2006, fueron visitadas 48 escuelas públicas, durante el cual se recolectó muestras de materia fecal de 388 niños y niñas entre 7 a 9 años escogidos aleatoriamente. Las muestras colectadas fueron examinadas por cuatro métodos para la búsqueda de enteroparásitos, el Directo en fresco, Faust, Ritchie and Lutz. El porcentaje de las muestras positivas fue de $94,23 \%$, donde fueron identificados siete tipos de protozoarios y cinco tipos de helmintos. La frecuencia de los enteroparásitos hallados en la población estudiada fue la siguiente: Giardia duodenalis 67\%, Entamoeba coli 52\%, Blastocystis hominis 45\%, Endolimax nana 31\%, Chilomastix mesnili $8 \%$, Iodamoeba butschilii 3\%, Entamoeba histolytica 2\%, Hymenolepis nana 12\%, Enterobius vermicularis 1\%, Strongyloides stercoralis 2\%, Ascaris lumbricoides $1 \%$ y Uncinarias $2 \%$.

Palabras clave: enteroparásitos, infecciones parasitarias, salud infantil.

\section{ABSTRACT}

Parasitic diseases have worldwide distribution and, despite the efforts of health organizations, remain a problem specially in developing countries like ours. The objective of this work, evaluated by the National Commission for Development of ethics in Paraguay, was to determine the frequency rate of enteroparasites in children of the first cycle of the basic education of public schools in Ciudad Del Este (Paraguay). In the period between September and December 2006, 48 public schools were visited and fecal samples of 388 children, who were between 7 and 9 years old and randomly selected, were collected. The collected samples were examined by four methods for entereoparasite search: Direct examination of fresh feces, Faust, Ritchie and Lutz. The percentage of positive samples was $94.23 \%$ and seven species of protozoans and five species of helminths were identified. The frequency of the enteroparasites in the population studied was as follows: Giardia duodenalis 67\%, Entamoeba coli 52\%, Blastocystis hominis 45\%, Endolimax nana 31\%, Chilomastix mesnili 8\%, Iodamoeba butschilii 3\%, Entamoeba histolytica 2\%, Hymenolepis nana 12\%, Enterobius vermicularis 1\%, Strongyloides stercoralis 2\%, Ascaris lumbricoides 1\% and Uncinarias 2\%.

Keywords: enteroparasites, school children, Ciudad del Este.

*Autor Correspondiente: Gloria Elena Cardozo Ocampos, Escuela de Posgrado. Facultad de Ciencias de la Salud, Universidad Nacional del Este. Paraguay

Email: gloricard4@gmail.com

Fecha de recepción: noviembre 2014; Fecha de aceptación: febrero 2015 


\section{INTRODUCCIÓN}

Las infecciones parasitarias intestinales tienen una distribución mundial, con tasas de frecuencias elevadas en numerosas regiones. Representan un indicador del estado ambiental, así como de las caracteristicas sociales, económicas y culturales de una población. Los enteroparásitos pueden llegar a ocasionar cuadros digestivos, que pueden tener severa repercusión sobre el crecimiento y desarrollo de los niños y las niñas. Durante largo tiempo pueden permanecer asintomáticos y sin ser diagnosticados, pero con igual riesgo sobre la salud de los mismos $(1,2)$.

A pesar de muchos esfuerzos por parte de los organismos de salud mundial para controlar estas enfermedades, no se tuvo una reducción de los índices de frecuencia. Ellas afectan a toda una familia, especialmente a aquellas de bajos ingresos, cuya condición de vida precaria, mala higiene y nutrición, contribuyen todavía más a la propagación de las enfermedades parasitarias $(1,2)$.

Varios trabajos demostraron que las tasas de frecuencia de parásitos son elevadas en América Latina y especialmente en países en vías de desarrollo como el nuestro. Dos trabajos hechos en Paraguay, concuerdan con los datos de publicaciones de países vecinos de que las enteroparasitosis constituyen un grave problema de salud pública, y según uno de estos, en el Paraguay, se presenta la misma realidad que afecta sobre todo a la población infantil. Numerosos factores se conjugan para establecer el panorama de esta problemática, entre los cuales se destacan la escasa cobertura de los servicios sanitarios en gran parte del territorio nacional, asi como las malas condiciones higiénicosanitarias y la poca educación de la población.

Es sabido que la epidemiología de las enteroparasitosis no puede ser bien estudiada, ya que es común que las personas afectadas no recurran a los servicios de salud por presentar este problema. Además, la metodología aplicada para la detección no siempre es la adecuada para diagnosticar los varios tipos de enteroparásitos, debiendo ser escogida muy bien según la sensibilidad de cada uno $(3,4)$.

Ciudad del Este es una ciudad del extremo este del Paraguay, capital del departamento de Alto Paraná, situada a 327 km de la capital del país, Asunción. Por su población y por su desarrollo económico, es la segunda ciudad más importante del Paraguay, contando con 312.652 habitantes. Forma parte de la zona conocida como Triple Frontera, en donde contacta la soberanía paraguaya con la del Brasil en Foz do Iguaçu, y con la soberanía de la Argentina en Puerto Iguazú. Es considerada una zona urbana (5).

El clima de Ciudad del Este es subtropical húmedo. La temperatura media anual es de $22^{\circ} \mathrm{C}$. El promedio anual de precipitaciones es abundante, con $1.850 \mathrm{~mm}$ aproximadamente. El verano es caluroso, con un promedio en enero de $27^{\circ} \mathrm{C}$. La elevada humedad suele volver sofocante al calor. El invierno es fresco, con una temperatura promedio de julio de $17^{\circ} \mathrm{C}$. Raramente se dan temperaturas inferiores a $1^{\circ} \mathrm{C}$ o superiores a $38^{\circ} \mathrm{C}$. Cuenta con servicios de agua potable y recolección de basura, pero que no llegan a toda la población. La mayor parte de la ciudad no cuenta con alcantarillado (5).

Las deficientes condiciones sanitarias (ambientales, de infraestructura y educación) predisponen a un mayor riesgo de infección por helmintos y protozoarios (6). Bajo el supuesto de que Ciudad del Este podría presentar estos problemas relacionados con las condiciones higiénicas del ambiente, el objetivo general del presente trabajo fue determinar la tasa de frecuencia de enteroparásitos en alumnos del primer ciclo de la Educación Escolar Básica de las Escuelas Públicas de este municipio.

La determinación del índice de enteroparasitosis y sus frecuencias constituyen instrumentos fundamentales para el diseño de las estrategias de higiene y educación sanitaria que pueden ayudar a disminuir este problema a través la prevención. 


\section{MATERIALES Y MÉTODOS}

Estudio descriptivo transversal, para lo cual fueron sorteadas 50 escuelas públicas de las 152 registradas en la base de datos del Ministerio de Educación y Cultura (7), y de cada una de ellas participarian 8 alumnos de ambos sexos entre 7 y 9 años escogidos aleatoriamente. Así el margen de error de la muestra sería de $\pm 5 \%$.

Finalmente, formaron parte de este proyecto 48 escuelas públicas, luego de la obtención del consentimiento informado de los padres o tutores de los niños, se trabajó con 388 muestras de materia fecal recolectadas en forma seriada de cinco dias en solución de formalina al $10 \%$, pertenecientes a alumnos escogidos aleatoriamente en cada escuela que participó del proyecto. Con esta cantidad de muestra se pudo trabajar con un margen de error de $\pm 4,9 \%$.

El diagnóstico de las enfermedades parasitarias suele ser complicado, debido a que muchos factores influyen, como ser el ciclo biológico del parásito y los métodos de detección de los mismos. El examen coproparasitoscópico es un conjunto de técnicas diagnósticas que constituyen la indicación para la identificación de la mayoría de las enteroparasitosis causadas por protozoarios o helmintos. Su eficacia y sensibilidad para establecer un diagnóstico correcto dependen de la adecuada indicación y preparación de la muestra, los datos clínicos y antecedentes de interés que sean aportados al laboratorio y de su correcta y completa ejecución con examen directo microscópico $(3,4,6)$.

Para una mejor detección de los parásitos, se optó por utilizar cuatro métodos de examen coproparasitológico. Así, las muestras fueron examinadas en el laboratorio de Parasitología de una Universidad de la región utilizando los métodos: Directo en fresco, Faust et al., Ritchie et al. y Lutz.

El examen directo en solución salina fisiológica y en solución de lugol, se basa en la utilización de solución salina fisiológica para conservar condiciones semejantes a las del cuerpo humano y de esta manera, es muy útil para reconocer trofozoítos de protozoarios y otros estadios de diagnóstico de helmintos y protozoarios. La solución de lugol permite colorear en forma temporal los trofozoítos y quistes e inmovilizar las larvas. Los resultados que arrojan falsos negativos o poco satisfactorios suelen deberse entre otros a: esperar mucho tiempo para el análisis, resecamiento de la preparación, muestras muy gruesas, y la carga parasitaria $(3,4)$.

Los métodos de concentración nos permiten aumentar la detección de parásitos debido a su mayor sensibilidad. El propósito es el de concentrar huevos de helmintos y quistes de protozoos cuando las infecciones son muy leves y no se detectan en preparaciones directas $(3,4)$.

El método de Faust se basa en la centrifugación-flotación utilizando sulfato de zinc obteniéndose un buena flotación de quiste y huevos. Las formas parasitarias son encontradas con facilidad, pues las preparaciones quedan con pocos artefactos. Entre sus desventajas, se pueden mencionar la deformación de los quistes de protozoos que dificulta su identificación y, la ausencia de flotación de los huevos infértiles de Ascaris No es el método adecuado para huevos de céstodos ni de tremátodos. Las larvas se encogen y no se puede reconocer su morfología específica $(3,4)$.

El método de Ritchie emplea éter y formaldehído y permite con el primero, liberar las formas parasitarias de las grasas, por disolución de las mismas y con el formol se fijan y conservan. La concentración se hace por centrifugaciones sucesivas. Algunas de las ventajas que tiene este método son concentrar y no deformar las formas parasitarias, permitir el transporte y almacenamiento de la materia fecal procesada antes de ser examinada. Se usa cuando se necesita una técnica para evaluación de tratamiento y determinación de frecuencia, cuando la excreción de quistes es baja e intermitente o para descartar infecciones leves en general, sobre todo si otros métodos no han ofrecido resultados esperados. Produce menos errores técnicos que otros métodos de concentración. Se debe tener cuidado con errores tales como que los huevos infértiles de 
Ascaris y en ocasiones quistes de Giardia pueden flotar y descartarse inadvertidamente con el tapón del tubo, no es adecuado para concentrar trofozoítos de protozoos $(3,4)$.

El método de Lutz o sedimentación espontánea es recomendado para concentrar huevos y larvas. Tiene como grandes ventajas su costo que es mucho menor y el menor riesgo para el personal de laboratorio mientras que su gran desventaja es el tiempo que se emplea $(3,4)$.

Los datos obtenidos fueron procesados con función estadística de Excel.

\section{RESULTADOS}

De las 388 muestras, 367 (94,23\%) fueron positivas para algún tipo de parásitos y 21 $(5,77 \%)$ fueron negativas. Se identificaron 12 géneros de enteroparásitos, de los cuales 7 eran protozoarios y 5 helmintos. En la Tabla 1 se presenta el número de muestras positivas para cada parásito detectado por método y en la Tabla 2 se contempla la frecuencia de cada uno de los parásitos identificados por cada uno de los cuatro métodos.

Tabla 1. Número de muestras de enteroparásitos según resultados positivos en exámenes por cuatro métodos coproparasitológicos en alumnos de las Escuelas Públicas de Ciudad del Este (Paraguay), 2006

\begin{tabular}{lccrr}
\hline Tipo / Parásito & \multicolumn{3}{c}{ Número de muestras positivas } & \\
& Directo en fresco & Faust & Ritchie & Lutz \\
\hline Protozoarios & & & & \\
Giardia lamblia & 228 & 198 & 231 & 246 \\
Entamoeba coli & 169 & 139 & 187 & 191 \\
Blastocystis hominis & 165 & 37 & 106 & 143 \\
Endolimax nana & 114 & 66 & 106 & 114 \\
Chilomastix mesnili & 29 & 22 & 22 & 27 \\
Iodamoeba butschilii & 7 & 0 & 7 & 11 \\
Entamoeba histolytica & 7 & 4 & 4 & 4 \\
Helmintos & 27 & 27 & 37 & 44 \\
Hymenolepis nana & 4 & 4 & 4 & 4 \\
Ascaris lumbricoides & 4 & 4 & 4 & 7 \\
Strongyloides stercoralis & 0 & 4 & 7 & 7 \\
Ancylostomideos & 0 & 0 & 4 & 0 \\
Enterobius vermicularis & & & & \\
\hline
\end{tabular}

Tabla 2. Frecuencia de enteroparásitos según resultados positivos en exámenes por cuatro métodos coproparasitológicos en alumnos de las Escuelas Públicas de Ciudad del Este

(Paraguay), 2006

\begin{tabular}{ccccc}
\hline Tipo / Parásito & \multicolumn{3}{c}{ Frecuencia (\%) por método } \\
& Directo en fresco & Faust & Ritchie & Lutz \\
\hline Protozoários & & & & \\
Giardia lamblia & $62 \%$ & $54 \%$ & $63 \%$ & $67 \%$ \\
Entamoeba coli & $46 \%$ & $38 \%$ & $51 \%$ & $52 \%$ \\
Blastocystis hominis & $45 \%$ & $10 \%$ & $29 \%$ & $39 \%$ \\
Endolimax nana & $31 \%$ & $18 \%$ & $29 \%$ & $31 \%$ \\
Chilomastix mesnili & $8 \%$ & $6 \%$ & $6 \%$ & $7 \%$ \\
Iodamoeba butschilii & $2 \%$ & $0 \%$ & $2 \%$ & $3 \%$ \\
Entamoeba histolytica & $2 \%$ & $1 \%$ & $1 \%$ & $1 \%$ \\
Helmintos & & & & \\
Hymenolepis nana & $7 \%$ & $7 \%$ & $10 \%$ & $12 \%$ \\
Ascaris lumbricoides & $1 \%$ & $1 \%$ & $1 \%$ & $1 \%$ \\
Strongyloides stercoralis & $1 \%$ & $1 \%$ & $1 \%$ & $2 \%$ \\
Ancylostomideos & $0 \%$ & $1 \%$ & $2 \%$ & $2 \%$ \\
Enterobius vermicularis & $0 \%$ & $0 \%$ & $1 \%$ & $0 \%$ \\
\hline
\end{tabular}


Como se puede observar, por cualquiera de los métodos, los protozoarios son los más frecuentes, siendo Giardia lamblia el que aparece en mayor porcentaje. Entre los helmintos, el más frecuente, por cualquiera de los métodos es Hymenolepis nana.

Por tres de los métodos (Directo en fresco, Ritchie y Lutz) se mantiene un orden de frecuencia de protozoarios empezando por el que se encuentra en mayor porcentaje Giardia lamblia, y en luego en orden descendente de porcentaje de frecuencia aparecen: Entamoeba coli, Blastocystis hominis, Endolimax nana, Chilomastix mesnili, Iodamoeba butschilii, Entamoeba histolytica. Solamente por el método de Faust se altera este orden, en donde Iodamoeba butschilii tuvo resultado negativo.

En el caso de los helmintos, tanto por el método Directo en fresco como por el de Faust, se obtuvo el siguiente orden de porcentajes de frecuencia descendente: Hymenolepis nana, Ascaris lumbricoides, Strongyloides stercoralis, Ancylostomideos y Enterobius vermicularis; mientras que por los métodos de Ritchie y de Lutz este orden se altera. En ambos caso, Hymenolepis nana sigue siendo el más frecuente mientras que en el método de Ritchie, el segundo más frecuente fue Ancylostomideos, seguido de Ascaris lumbricoides, Strongyloides stercoralis y Enterobius vermicularis. En el método de Lutz, los segundos más frecuente fueron Strongyloides stercoralis y Ancylostomideos, seguidos de Ascaris lumbricoides.

Las asociaciones también aparecieron, pero no fueron incluidas en este trabajo.

El porcentaje de cada uno de los parásitos no fue hallado por metodología específica, por lo que se tomó como frecuencia adecuada aquel resultado que fue obtenido por el método en el que se ha encontrado mayor porcentaje para cada parásito detectado. Sobre la base de este criterio, se concluye que la frecuencia de los parásitos encontrados en la población estudiada sería como se presenta en la Tabla 3.

Tabla 3. Frecuencia de enteroparasitos según resultados positivos en exámenes realizados a alumnos de las Escuelas Públicas de Ciudad del Este (Paraguay), 2006

\begin{tabular}{llll}
\hline \multicolumn{1}{c}{ Parasito } & Frecuencia & Método \\
\hline Protozoários & & \\
& Giardia lamblia & $67 \%$ & Lutz \\
& Entamoeba coli. & $52 \%$ & Lutz \\
Blastocystis hominis & $45 \%$ & Directo en fresco \\
& Endolimax nana & $31 \%$ & Directo en fresco o Lutz \\
& Chilomastix mesnili & $8 \%$ & Directo en fresco \\
& Iodamoeba butschilii & $3 \%$ & Lutz \\
Entamoeba histolytica & $2 \%$ & Directo en fresco \\
& & & \\
& Hymenolepis nana & $12 \%$ & Lutz \\
& Enterobius vermicularis & $1 \%$ & Ritchie \\
& Strongyloides stercoralis & $2 \%$ & Lutz \\
& Ascaris lumbricoides & $1 \%$ & Todos los métodos \\
& & & utilizados \\
& Ancylostomideos & $2 \%$ & Ritchie o Lutz
\end{tabular}

\section{DISCUSIÓN}

Los resultados obtenidos en este trabajo coinciden con otros realizados en el país y en otros países de Sudamérica. Según Salomón (2.005), los protozoos son los parásitos más frecuentes especialmente en niños menores de 11 años (8). Igualmente, varios autores refieren que el porcentaje de protozoarios supera al de los helmintos, así como la identificación de los mismos parásitos como ser: Blastocystis hominis, Giardia lamblia, 
Endolimax nana, Entamoeba coli, Chilomastix mesnilii, Enterobius vermicularis e Hymenolepis nana (8-14).

En Paraguay, tanto Zunini (2.001) como Jure (2.003) plantean que el porcentaje de los protozoarios supera al de los helmintos, además también coinciden en que el que aparece con mayor frecuencia es Giardia lamblia. Así según Canese, el porcentaje de este protozoario fue de $67 \%$, y según Zunini fue de $42 \%$. Esto se da utilizando métodos iguales a los de este trabajo o diferentes, en el caso de Zunini, utilizó el método de KatoKatz $(9,10)$.

En Perú, según Cabrera (2.000) el porcentaje no coincide, ya que este autor refiere $10,57 \%$ de Giardia lamblia. También para los otros protozoarios existen diferencias, así por ejemplo este mismo autor informa más de $60 \%$ de Entamoeba coli, que aquí, por cualquiera de los métodos no alcanza ese porcentaje, siendo el máximo obtenido de $52 \%$ (11). Sin embargo, Iannacone (2.006) sostiene que Giardia es considerado el protozoario patógeno más frecuente en la población infantil en el Perú y de registro obligatorio en toda la encuesta parasitológica, y menciona que su frecuencia está entre 10 y $80 \%$ (12).

Iannacone (2.006) sostiene que Blastocystis hominis, es un protozoo que cada vez adquiere mayor importancia, su prevalencia ha alcanzado el $70 \%$ en Perú, y este fenómeno de incremento se ha observado en otros países sudamericanos como Argentina, Chile y Venezuela $(8,12-14)$. En este trabajo, los denominados comensales también presentan una frecuencia mayor, es por ello que no se debe restar importancia a la frecuencia hallada para Blastocystis hominis, hasta $45 \%$, ya que es indicativa de las condiciones higiénicas en las que vive la población, ya sea en el ambiente escolar o familiar.

Con respecto a los helmintos, según Zunini (2.001) y utilizando el método de Kato-Katz, el porcetaje de Hymenolepis nana fue de 12,8\%, también considerando el más frecuente comparado con otros helmintos (9).

Para los helmintos, existen métodos más recomendados según el tipo comparado con los que incluyen el estudio coproparasitologico, por ejemplo hisopado anal para Enterobius vermicularis o cultivo de heces para Strongyloides. Esto podría explicar la frecuencia baja encontrada en este trabajo comparada con las de los protozoarios $(3,4)$.

La patogenia de la mayoría de los parásitos que fueron encontrados no es simple, pueden causar problemas graves de salud, especialmente en la población infantil. La educación sanitaria y la mejora de las condiciones de saneamiento de la ciudad podrían evitar nuevas infecciones.

Sobre la base de lo expuesto, con los datos obtenidos en este trabajo se espera contribuir en la toma de decisiones en lo que respecta a salud pública, medio ambiente y educación.

\section{REFERENCIAS BIBLIOGRÁFICAS}

1. Rey L. Parasitología: Parásitos e doenças parasitarias do homem nas Américas e na África. $2^{a}$ ed. Rio de Janeiro: Guanabara - Koogan, 1991.

2. Cimerman S, Cimerman B, Lewi DS. Parasitoses intestinais: Visão critica de sua importância em nosso meio. Ars Curandi. 1998; 31:5-9.

3. De Carli GA. Parasitología clínica: Seleção de métodos e técnicas de laboratório para o diagnostico das parasitoses humanas. Sao Paulo: Atheneu; 2001.

4. Girard de Kaminsky R. Huevos y larvas de Helmintos; ooquistes y quistes de protozoos: Concentración por Formalina - Acetato de Etilo. En: Manual de parasitología: Métodos para laboratorios de atención primaria de salud [Internet]. $2^{\circ}$ ed. Nueva Orleans: OPS, OMS, Universidad Nacional Autònoma de Honduras; 2003. p. 43-45. [acceso 5 de noviembre 2014]. Disponible en: http://www.bvs.hn/Honduras/pdf/Manual\%20Parasitologia\%202007.pdf

5. Soriano SV, Manacorda AM, Pierangeli NB, Navarro MC, Giayetto A, Barbieri LM, et al. Parasitosis intestinales y su relación con factores socioeconómicos y condiciones de hábitat en niños de Neuquén, Patagonia, Argentina. Parasitol Latinoam. 2005; 60(3-4):154-61. 
6. Salomon MC, Tonelli RL, Borremans CG, Bertello D, De Jong LI, Jofre CA, et al. Prevalencia de parásitos intestinales en niños de la ciudad de Mendoza, Argentina. Parasitol Latinoam. 2007; 62:49-53.

7. Zunini M, Nuñez MT. Prevalencia de las parasitosis intestinal en escolares a nivel de la comunidad. LILACS. 2001: 9.

8. Jure D, Muñoz M, Canese J, Canese A. Prevalencia de parásitos intestinales en niños escolares de Paraguay. Rev. Parag Microb. 2003; 23(1): 52-7.

9. Cabrera M, Verastegui M, Cabrera R. Prevalencia de enteroparásitos en una comunidad altoandina de la Provincia de Victor Fajardo, Ayacucho, Perú. Rev gastroenterol Perú [internet].2005 [acceso 8 de noviembre 2014]; 25(2):150-5. Disponible en:

http://www.scielo.org.pe/scielo.php?script=sci_arttext\&pid=S1022-51292005000200003

10. Iannacone J, Benites MJ, Chirinos L. Prevalencia de infección por parásitos intestinales en escolares de primaria de Santiago de Surco, Lima, Perú. Parasitol Latinoam. 2006; 61(1-2):54-62.

11. Michelli E, De Donato M. Prevalencia de Blastocystis hominis en habitantes de Río Caribe, Estado de Sucre, Venezuela. Saber Univ de Oriente Ven. 2001; 13(2):105-12.

12. Mercado R, Castillo D, Muñoz V. Enteroparasitosis en escolares de la comuna de Colina, Región Metropolitana, Santiago, Chile. 2003. En: Libro de Resúmenes del $6^{\circ}$ Congreso Latinoamericano de Parasitología; 2003 oct 1 al 3; La Paz- Bolivia. p.73.

13. Costa-Macedo LM, Machado-Silva JR, Rodriguez-Silva R, Oliveira LM, Ripper Vianna MS. Enteroparasitoses em pre-escolares de comunidade favelizadas da cidade do Rio de Janeiro, Brasil. Cad. Saude Pública. 1998; 14(4): 851-5.

14. Acuña AM, Da Rosa D, Colombo $H$, Salomón $S$, Alfonso A, Combol A, et al. Parasitosis intestinales en guarderías comunitarias de Montevideo. Rev. Med. Uruguay. 1999; 15(I): 24-33. 